\title{
Evidence of Singlet-Triplet Crossing in Photoluminescence of Positively Charged Excitons in GaAs Quantum Wells
}

\author{
L. Bryja, A. Wójs, J. Jadczak, J. Misiewicz \\ Institute of Physics, Wrocław University of Technology \\ Wybrzeże Wyspiańskiego 27, 50-370 Wrocław, Poland \\ P. PŁochocka, M. Potemski \\ Grenoble High Magnetic Field Laboratory, CNRS \\ 25 av. Martyrs, F-38042 Grenoble, France \\ D. Reuter And A. Wieck \\ Lehrstuhl fur Festkörperphysik, Ruhr Universität \\ Universitätstrasse 150, 44780 Bochum, Germany
}

\begin{abstract}
Positively charged excitons in a two-dimensional hole gas in symmetric and asymmetric $\mathrm{GaAs} / \mathrm{Ga}_{1-x} \mathrm{Al}_{x} \mathrm{As}$ quantum wells are studied in polarization-resolved photoluminescence experiments in high magnetic fields $B$ (up to $23 \mathrm{~T}$ ) and low temperatures (down to $300 \mathrm{mK}$ ). The experiments are accompanied by numerical calculations of a real structure. The whole family of trions (the singlet and a pair of triplets) are observed. The Coulomb energies crossing of singlet and triplet is found: hidden in symmetric and visible in asymmetric structures.
\end{abstract}

PACS numbers: 73.20.Mf, 71.35.Ji, 71.35.Pq, 78.20.Ls

\section{Introduction}

The two-dimensional (2D) gases composed of electrons or holes are very unique systems for investigation of many-body interactions [1]. In the last years the negatively charged excitons (negative trions) in 2D electron gas have been studied very intensively, while the positively charged excitons (positive trions) in $2 \mathrm{D}$ hole gas are considerably less explored. In a good quality low-dimensional semiconductor nanostructures excitons and trions often determine the photoluminescence $(\mathrm{PL})$ spectra $[2,3]$. In $2 \mathrm{D}$ quantum wells subjected to high magnetic 
fields $B$, the trion energy spectrum contains the following bound states labeled by the pair spin $S$ and relative angular momentum $M[4,5]$ : spin-singlet with $M=0$ $\left(\mathrm{X}_{\mathrm{s}}^{ \pm}\right)$and a pair of triplets with $M=-1\left(\mathrm{X}_{\mathrm{td}}^{ \pm}\right)$and $M=0\left(\mathrm{X}_{\mathrm{tb}}^{ \pm}\right)$. Only the $M=0$ states are optically active ("bright"); while recombination of states with $M \neq 0$ ("dark") requires breaking of the $2 \mathrm{D}$ translational invariance (e.g., by well width fluctuations or impurities).

We report on the studies of positively charged excitons in symmetric and asymmetric GaAs/ $\mathrm{Ga}_{1-x} \mathrm{Al}_{x}$ As quantum wells with a two-dimensional hole gas. We performed polarization resolved PL measurements accompanied with transport experiments in high magnetic fields (up to $B=23 \mathrm{~T}$ ) and at low temperatures (down to $T=300 \mathrm{mK}$ ). Experimental PL and transport studies were supplemented with realistic configuration-interaction calculations. We report on the experimental detection of the entire family of positively charged excitons: the pair of bright and dark triplets and the singlet. We found crossing of the singlet and triplet Coulomb energies: hidden in symmetric and visible in asymmetric structures.

\section{Experiment, results, and discussion}

The studied samples were $\mathrm{GaAs} / \mathrm{Ga}_{0.65} \mathrm{Al}_{0.35} \mathrm{Al}$ quantum wells fabricated by molecular beam epitaxy on a (001) semi-insulating GaAs substrate and $\delta$ doped with carbon in the barriers. We studied two types of samples. The symmetric structure was a $15 \mathrm{~nm}$ wide GaAs/AlGaAs quantum well, symmetrically carbon delta-doped in both barriers, with the 2D hole concentration (measured in dark) $p=1.15 \times 10^{11} / \mathrm{cm}^{-2}$ and mobility $\mu=1.01 \times 10^{5} \mathrm{~cm}^{2} /(\mathrm{V} \mathrm{s})$. The asymmetric structure was $22 \mathrm{~nm}$ wide GaAs/AlGaAs quantum well, carbon delta-doped on one side, with $2 \mathrm{D}$ dark concentration $p=1.8 \times 10^{11} / \mathrm{cm}^{-2}$ and mobility $\mu=1.2 \times 10^{5} \mathrm{~cm}^{2} /(\mathrm{V} \mathrm{s})$. From the measurements of longitudinal and Hall resistance, performed under the same experimental conditions as PL, we determined the actual 2D hole gas concentration under illumination, and found the concentration decrease to the extent dependent on the density of light. The PL measurements were carried out at low temperatures from $T=300 \mathrm{mK}$ to $T=1.8 \mathrm{~K}$ and in high magnetic fields up to $B=23 \mathrm{~T}$ applied perpendicularly to the structure. The fiber glass optics was applied. Experiments were performed in the Faraday configuration, with a linear polarizer and wave quarter placed in the liquid helium close to the sample. To switch between the $\sigma^{-}$and $\sigma^{+}$polarizations, the direction of magnetic field was changed. PL was excited by the $\lambda=514 \mathrm{~nm}$ line of argon ion laser.

When the laser power density is low, below $P<10 \mathrm{~mW} / \mathrm{cm}^{2}$, in the PL spectra of all studied samples in zero magnetic field one line attributed to radiative recombination of photoexcited electrons with $2 \mathrm{D}$ holes is observed. In the studied samples, the recombining exciton species are positively charged excitons, it is they consist of 2 holes and one electron bound by the Coulomb interaction. According to theoretical calculation at $B=0$ the holes are in the singlet state. When magnetic field is applied, the PL spectra develop and reveal much richer structure. 


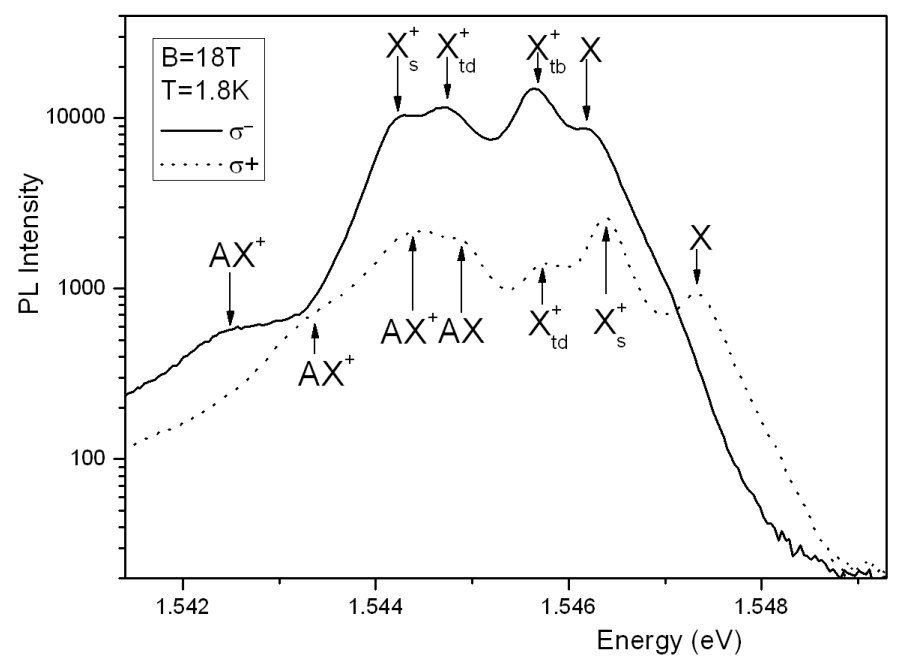

Fig. 1. Photoluminescence spectra for symmetric $15 \mathrm{~nm}$ QW in magnetic field $B=$ $18 \mathrm{~T}$.

In Fig. 1 the PL spectra for symmetric structure in both $\sigma^{ \pm}$are presented. From the detailed analysis of the evolution of PL spectra as a function of the magnetic field (not presented) and comparison of experimental data of the Coulomb binding energies in the trion complex with realistic numerical calculations we identified all observed lines (for more detailed explanation see Ref. [6]). The order of energy positions of the trion lines in respect of $\mathrm{X}$ is different in both polarizations. In $\sigma^{-}$descending energy from $\mathrm{X}$ we detect: "bright triplet" $\mathrm{X}_{\mathrm{tb}}^{+}$, "dark triplet" $\mathrm{X}_{\mathrm{td}}^{+}$and finally the singlet $\mathrm{X}_{\mathrm{s}}^{+}$. In $\sigma^{+}$the order of singlet and dark triplet lines is reversed. The bright triplet line is not observed in this polarization. We also observe radiative recombination of excitons bound to neutral and positively charged acceptor $-\mathrm{AX}$ and $\mathrm{A}^{+} \mathrm{X}$ (i.e., $\mathrm{AX}^{+}$).

From the comparison of $\sigma^{+}$and $\sigma^{-}$spectra we also extracted the Zeeman splitting for the recombination of different states. Interestingly, they are nearly identical for $\mathrm{X}$ and $\mathrm{X}_{\mathrm{td}}^{+}$, considerably lower than for $\mathrm{X}_{\mathrm{S}}^{+}$or $\mathrm{AX}^{+}$(not presented). This is traced to the $k$-dependence of the hole Lande $g$-factor. Let us note that it causes reversal of the order of $\mathrm{X}_{\mathrm{td}}^{+}$and $\mathrm{X}_{\mathrm{s}}^{+}$peaks in the $\sigma^{+}$and $\sigma^{-}$spectra. To remove the Zeeman contribution from the trion binding energy, the exciton $(\mathrm{X})$ and trion $\left(\mathrm{X}^{+}\right)$peak positions measured in PL must be averaged over both $\left(\sigma^{ \pm}\right)$ polarizations. The resulting curves of $\mathrm{X}_{\mathrm{s}}^{+}$and $\mathrm{X}_{\mathrm{td}}^{+}$are displayed in Fig. 2. The results of realistic numerical calculations are also plotted. A singlet-triplet crossing is found at $B \approx 12 \mathrm{~T}$ (relatively low field compared to the crossing of negative trions in typical $n$-doped wells). Due to the strong difference in the Zeeman splitting this crossing is hidden in PL spectra. Let us notice that nevertheless the numerical calculations give a slightly lower binding energy for the additional hole, the singlet-triplet crossing is obtained almost in the same magnetic field. 


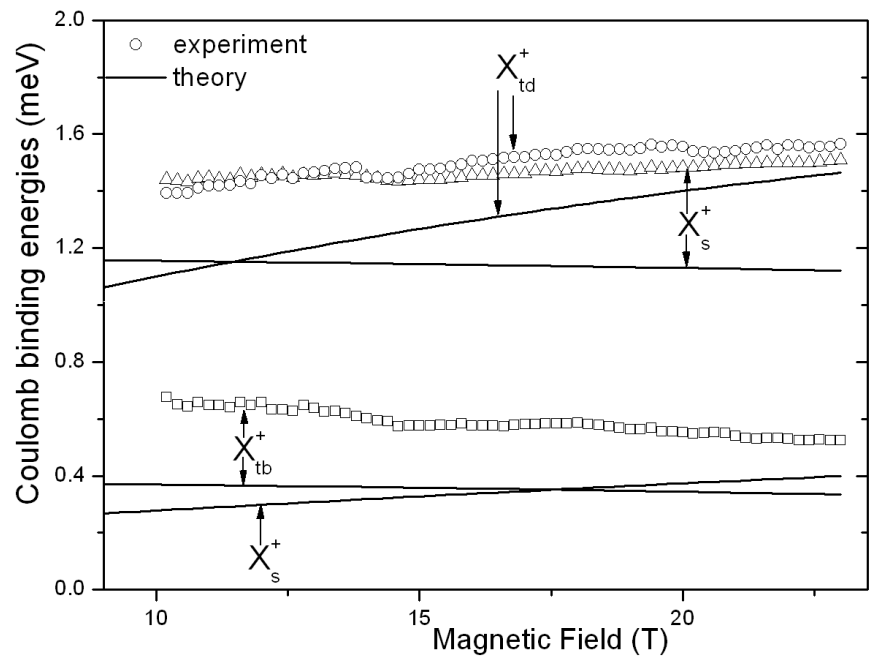

Fig. 2. The second-hole Coulomb binding energies: symbols - experiment, solid lines calculated numerically for the $15 \mathrm{~nm}$ symmetric GaAs quantum well.

In the evolution of PL spectra for the asymmetric $22 \mathrm{~nm} \mathrm{QW}$ in $\sigma^{-}$polarization in the magnetic field from $B=11$ to $16 \mathrm{~T}$ (not shown), we clearly see the crossing of two lines at $B \approx 13.5 \mathrm{~T}$. From the comparison of experimental data of the Coulomb binding energies in the trion complex with numerical calculations and detailed analysis of PL spectra evolution in magnetic field we assess this feature to a visible singlet triplet crossing.

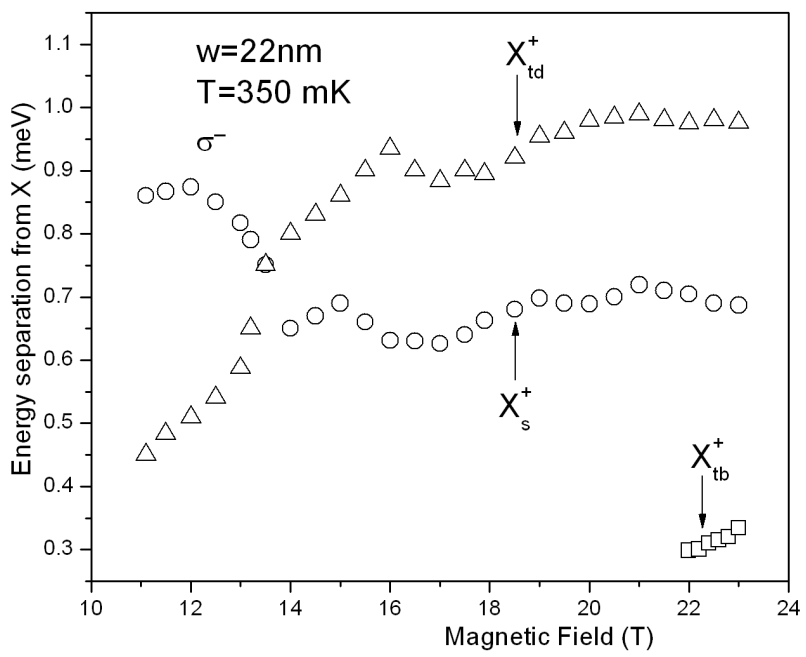

Fig. 3. The experimental second-hole Coulomb binding energies for the $22 \mathrm{~nm}$ asymmetric GaAs quantum well. 
In Fig. 3 the energy distance of singlet $\mathrm{X}_{\mathrm{s}}^{+}$and triplet $\mathrm{X}_{\mathrm{td}}^{+}$lines from excitons are shown as a function of $B$. These energies agree well with theoretical calculations of the Coulomb binding energies of additional hole in the asymmetric sample with lower concentration which satisfied experimental conditions. Due to high density of laser power excitation we decreased strongly the 2D hole concentration to $p=1.23 \times 10^{11} / \mathrm{cm}^{-2}$.

\section{Acknowledgments}

Work supported by grants RITA-CT-2003-505474 from EC and by grants N202-071-32/1513 and N202-104-31/0771 from Polish MNiSW.

\section{References}

[1] D.C. Tsui, H.L. Stormer, A.C. Gossard, Phys. Rev. Lett. 48, 1559 (1982).

[2] K. Kheng, R.T. Cox, M.Y. d'Aubigné, F. Bassani, K. Saminadayar, S. Tatarenko, Phys. Rev. Lett. 71, 1752 (1993).

[3] M. Byszewski, B. Chwalisz, D.K. Maude, M.L. Sadowski, M. Potemski, T. Saku, Y. Hirayama, S. Studenikin, D. G. Austing, A.S. Sachrajda, P. Hawrylak, Nature Phys. (London) 2, 239 (2006).

[4] A. Wójs, J.J. Quinn, P. Hawrylak, Phys. Rev. B 62, 4630 (2000).

[5] G. Yusa, H. Shtrikman, I. Bar-Joseph, Phys. Rev. Lett. 87, 216402 (2001).

[6] L. Bryja, A. Wójs, J. Misiewicz, M. Potemski, D. Reuter, A. Wieck, Phys. Rev. B 75, 035308 (2007); A. Wójs, Phys. Rev. B 76, 085344 (2007). 